\title{
Bundesamt für Statistik, so nicht!
}

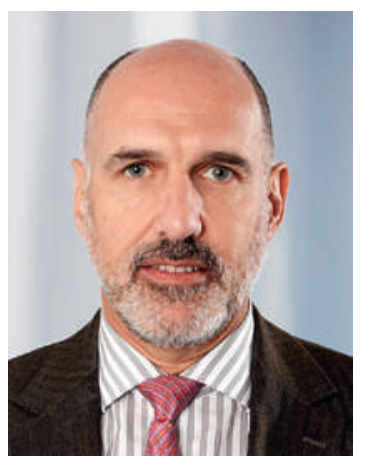

Wie auf Seite 1115 dieser Ausgabe der Schweizerischen Ärztezeitung nachzulesen ist, stürzt sich das Bundesamt für Statistik (BFS) mit Feuereifer auf die Erhebung von Daten über die Tätigkeit von Ärzten und anderen Pflegefachleuten in der Schweiz.

Keine gesetzliche Grundlage? Aber das ist doch kein Problem! Das übergeordnete Interesse der BFS-Datenbanken hat schliesslich Vorrang vor untergeordneten juristischen oder ethischen Bedenken ... und beim Arzt- bzw. Patientengeheimnis handelt es sich ja offenkundig um einen veralteten Begriff, überholt, vollkommen unnötig!

Worum geht es? Artikel 22a KVG [1] regelt die Übermittlung von Daten aus den Praxen an das BFS zum Zwecke der «Anwendung der Bestimmungen dieses Gesetzes über die Wirtschaftlichkeit und Qualität der Leistungen». Bei den geforderten Daten handelt es sich unter anderem um die «Anzahl und Struktur der Patientinnen und Patienten in anonymisierter Form».

Dieses Gesetz ist bereits seit dem 1. Januar 2009 in Kraft und muss nun endlich umgesetzt werden. Aber nicht auf eigene Faust!

\section{Dieses Projekt ist in Bezug auf das Arztgeheimnis ein echter Skandal, ein eklatanter Widerspruch zum Gesetz und ein vollkommen absurder Plan im Hin- blick auf den enormen Verwaltungsaufwand für die Praxen.}

Das Bundesamt für Statistik beharrt bei der Umsetzung dieser Bestimmungen nämlich darauf, sämtliche Informationen über alle Patienten und alle Untersuchungen in allen Arztpraxen des Landes zu erfassen - und zwar nicht in wirklich anonymisierter Form, da diese Daten letztendlich dazu dienen sollen, für jeden Menschen in der Schweiz einen «Gesundheitslebenslauf» zu erstellen. Daher ist dieses Projekt in Bezug auf das Arztgeheimnis ein echter Skandal, ein eklatanter Widerspruch zu einem Gesetz, das lediglich die Erfassung von «Anzahl und Struktur der Patientinnen und Patienten in anonymisierter Form» erlaubt, und ausserdem ein vollkommen absurder Plan im Hinblick auf den enormen Verwaltungsaufwand für die Praxen.

Natürlich wird sich die FMH sowohl im Namen der Ärzteschaft als auch der fundamentalen Grundsätze der Medizin diesem MARS-Projekt (so lautet seine Bezeichnung) des BFS erbittert widersetzen. Wir werden die Datenerfassung der gesamten Bevölkerung, die Instrumentalisierung der Ärzteschaft und die gesetzeswidrigen Machenschaften eines Bundesamtes nicht einfach hinnehmen.

Abgesehen davon bietet uns dieser Heisshunger der Bundesverwaltung auf Daten die Gelegenheit, noch einmal zu erläutern, warum das Arztgeheimnis so wichtig ist.

\section{Ein derart zentrales Element wie die Medizin darf in einer menschlichen Gesellschaft nicht ungestraft beiseite- geschoben oder missbraucht werden.}

Natürlich bietet der rasante technische Fortschritt nahezu uneingeschränkte Möglichkeiten im Hinblick auf die Speicherung von Daten und die Überwachung von Personen, unabhängig davon, ob es sich im Einzelfall um Ärzte oder Patienten handelt. Dies ist aber noch lange kein Grund, wirklich alles umzusetzen! Vielleicht müsste an dieser Stelle daran erinnert werden, dass es nicht sehr intelligent ist, auf der Strasse so schnell zu fahren, wie es technisch möglich wäre, alle Entdeckungen der Nuklearphysik tatsächlich in die Tat umzusetzen oder alles auszuschöpfen, was medizinisch machbar ist!

Jede Entdeckung, jeder wissenschaftliche oder technologische Fortschritt, jede neue Möglichkeit erfordert einen Augenblick des Nachdenkens, um sich ein Urteil zu bilden. Alles zu tun, «weil man es kann», erscheint doch recht kindisch.

Und als weitere, noch subjektivere Überlegung darf man nicht vergessen, dass die Medizin in der menschlichen Gesellschaft eine Geschichte hat, eine Substanz, ihren eigenen Platz, der nicht nur Pillen und Zäpfchen beinhaltet: Die Medizin ist ein zentrales Element jeder Gesellschaft, so auch unserer, in der sie eine ganz besondere Rolle spielt, bei der es im Wesentlichen um die Würde des Menschen und das Vertrauen, d. h. die ärztliche Schweigepflicht, geht.

Hier auf den Eid des Hippokrates zu verweisen, ist fast schon banal. Dieser 2500 Jahre alte Text beweist in seinem der Schweigepflicht gewidmeten Teil jedoch eindeutig, dass es sich hier nicht um eine zeitgenössische Marotte handelt, sondern um das Vertrauen des Patienten zum Arzt, mit dem er nicht nur über Fieber und Pusteln redet, sondern auch über sich selbst, sein Dasein, seine Lebensfragen und Unzulänglichkeiten. Das Vertrauen, welches das Arztgeheimnis bzw. die ärztliche Schweigepflicht ermöglicht, ist in der täglichen Praxis Grundvoraussetzung für Therapie und Heilung. Uns Ärztinnen und Ärzten obliegt es, diese Art von Medizin zu verteidigen ... und somit die Verantwortlichen in Politik und Verwaltung wieder und wieder daran zu erinnern, dass ein derart zentrales Element wie die Medizin in einer menschlichen Gesellschaft nicht ungestraft beiseitegeschoben oder missbraucht werden darf.

Dr. med. Jacques de Haller, Präsident der FMH

1 www.admin.ch/ch/d/sr/832_10/index.html 